\title{
IMPLICATIONS OF A HIGGS INTERPRETATION OF THE $\zeta(8.3)^{*}$
}

\author{
Howard E. HABER
}

Department of Physics, University of California, Santa Cruz, CA 95064, USA and Standard Linear Accelerator Center, Theory Group - Bin 81, PO Box 4349, Stanford, CA 94305, USA

\section{G.L. KANE}

Randall Laboratory of Physics, University of Michigan, Ann Arbor, MI 48109, USA

Received 8 October 1984

We consider the implications if the new state seen by the Crystal Ball collaboration in radiative $r$-decay, the $\zeta(8.3)$, is interpreted as a Higgs boson. We show that $\mathrm{BR}(\zeta \rightarrow g g)<0.06$ or $\zeta(8.3)$ would already have been observed at FNAL. As a consequence, there would be very significant constraints on models which go beyond the standard model (supersymmetry, composite models, technicolor, ...). In particular, the minimal two-higgs doublet low-energy supersymmetric models would be ruled out. We give a sum rule restricting the number of heavy colored states which couple to $\zeta(8.3)$; remarkably, only one further generation of heavy quarks could exist. It is crucial to detect the prompt semileptonic decays from charm to confirm a Higgs interpretation.

\section{Introduction}

The Crystal Ball collaboration has recently announced a new state [1], $\zeta(8.3)$, which is seen in radiative $\boldsymbol{T}$-decay. The relevant data are as follows:

$$
\begin{gathered}
M(\zeta)=8.322 \pm 0.026 \mathrm{GeV}, \\
\Gamma(\zeta)<80 \mathrm{MeV} \quad(90 \% \mathrm{CL}), \\
\operatorname{BR}(\Upsilon \rightarrow \gamma \zeta)=(5.7 \pm 2.8) \times 10^{-3}, \\
\operatorname{BR}\left(\Upsilon \rightarrow \gamma \zeta, \zeta \rightarrow \tau^{+} \tau^{-}\right)<2 \times 10^{-3}, \\
\frac{\operatorname{BR}\left(\Upsilon^{\prime} \rightarrow \gamma \zeta\right)}{\operatorname{BR}(\Upsilon \rightarrow \gamma \zeta)}<0.22 \quad(90 \% \mathrm{CL}) .
\end{gathered}
$$

* Research supported in part by the US Department of Energy, contracts DE-AC02-76ER01112 and DE-AC03-76SF2515 and by the National Science Foundation, grant PHY-8115541. 
In addition, the $\zeta(8.3)$ is seen in both "high" and "low" multiplicity hadron samples; the latter may be a hint for the presence of the decay $\zeta \rightarrow \tau^{+} \tau^{-}$.

We are aware of no reasonable hadronic interpretation for the $\zeta(8.3)$ (with the possible exception of ref. [2]). Therefore, it seems natural to try to interpret this state as a Higgs boson [3-5]. In the minimal version of the standard electroweak model, containing one Higgs doublet, one expects the decay $\Upsilon \rightarrow \mathrm{H}^{0} \gamma$ to occur if kinematically allowed. The expected rate was first computed by Wilczek [6]; for $m_{\mathrm{H}}=8.3$ $\mathrm{GeV}$, one finds $\mathrm{BR}\left(T \rightarrow \gamma \mathrm{H}^{0}\right) \approx 5 \times 10^{\circ 5}$ which is two orders of magnitude too small (cf. eq. (1c)). However, in any electroweak model with more than one Higgs doublet, there are additional parameters ${ }^{\star}$ which can result in an enhancement (or suppression) of the $\mathbf{H}^{0} \mathrm{f} \overline{\mathrm{f}}$ coupling ( $\mathrm{f}=$ quark and/or lepton). Thus the observed rate in eq. (1c) cannot by itself rule out a Higgs interpretation for $\zeta(8.3)$.

\section{Two-doublet Higgs models}

We focus on a model consisting of two higgs doublets, $\phi_{1}$ and $\phi_{2}$ (each with hypercharge +1$)$. The two neutral components acquire vacuum expectation values $v_{1}$ and $v_{2}$. After symmetry breaking, one is left with five physical Higgs particles - two charged and three neutral bosons. If no $C P$ violation is introduced in the Higgs sector, then the neutral Higgs bosons conserve $C$ and $P$ separately in their interactions with fermions. Of the three neutral scalars, two are $J^{P C}=0^{-+}$ bosons and one is a $0^{-+}$boson. There are two parameters relevant to the $\mathrm{H}^{0} \mathrm{f} \bar{f}$ interactions. If $\left\langle\phi_{i}^{0}\right\rangle=v_{i}$, we can rotate our Higgs fields such that

$$
\begin{aligned}
& \chi_{1}=\phi_{1} \cos \beta+\phi_{2} \sin \beta, \\
& \chi_{2}=-\phi_{1} \sin \beta+\phi_{2} \cos \beta, \quad \tan \beta=v_{2} / v_{1}
\end{aligned}
$$

(we normalize $v_{1}^{2}+v_{2}^{2}=2 \mathrm{~m}_{\mathrm{w}}^{2} / \mathrm{g}^{2} \approx 250 \mathrm{GeV}$ ). Then, $\left\langle\chi_{2}^{0}\right\rangle=0$ and we may identify the physical Higgs bosons as $H^{ \pm} \equiv \chi_{2}^{\dagger}, H_{3}^{0} \equiv \sqrt{2} \operatorname{Im} \chi_{2}^{0}$ (pseudoscalar) and a linear combination of $\sqrt{2} \operatorname{Re} \chi_{2}^{0}$ and $\sqrt{2} \operatorname{Re}\left[\chi_{1}^{0}-\left(v_{1}^{2}+v_{2}^{2}\right)^{1 / 2}\right]$ (scalars). The appropriate linear combination of scalar Higgs bosons is determined by the Higgs mass matrix (which depends on parameters of the Higgs potential), and can be parameterized by an angle $\alpha^{\star \star}$ :

$$
\begin{aligned}
& H_{1}^{0}=\cos (\alpha-\beta)\left[\sqrt{2} \operatorname{Re} \chi_{1}^{0}-\sqrt{v_{1}^{2}+v_{2}^{2}}\right]+\sin (\alpha-\beta) \sqrt{2} \operatorname{Re} \chi_{2}^{0}, \\
& H_{2}^{0}=-\sin (\alpha-\beta)\left[\sqrt{2} \operatorname{Re} \chi_{1}^{0}-\sqrt{v_{1}^{2}+v_{2}^{2}}\right]+\cos (\alpha-\beta) \sqrt{2} \operatorname{Re} \chi_{2}^{0} .
\end{aligned}
$$

\footnotetext{
- The possibility of a uniform enhancement of all $\mathrm{H}^{0} \mathrm{f} \overline{\mathrm{f}}$ couplings was first mentioned by Wilczek in ref. [6]. It was independently proposed and studied in detail in ref. [7].

* In the most general two-higgs doublet model, there are scven independent parameters - four Higgs boson masses, the angles $\alpha$ and $\beta$ and a possible $C P$ violating phase. (For simplicity, we have set the latter phase to zero in this paper.) Some of these parameters can be related if restrictions are imposed on the theory (such as a Peccei-Quinn symmetry or supersymmetry).
} 


\section{TABLE 1}

Coupling of fermions to neutral Higgs bosons are given by $\mathrm{gm}_{\mathrm{f}} /\left(2 \mathrm{~m}_{\mathrm{W}}\right)$ multiplicd by the factor given below (where $\sqrt{\frac{1}{2}} G_{\mathrm{F}} \equiv g^{2} / 8 m_{\mathrm{W}}^{2}$ )

\begin{tabular}{cccccc}
\hline & \multicolumn{2}{c}{ Model I } & & \multicolumn{2}{c}{ Model II } \\
\cline { 2 - 3 } & $\mathrm{u}$ & $\mathrm{d}$ & & $\mathrm{u}$ & $\mathrm{d}$ \\
\hline$H_{1}^{0}$ & $\frac{\cos \alpha}{\cos \beta}$ & $\frac{\cos \alpha}{\cos \beta}$ & & $\frac{\sin \alpha}{\sin \beta}$ & $\frac{\cos \alpha}{\cos \beta}$ \\
$H_{2}^{0}$ & $\frac{-\sin \alpha}{\cos \beta}$ & $\frac{-\sin \alpha}{\cos \beta}$ & & $\frac{\cos \alpha}{\sin \beta}$ & $\frac{-\sin \alpha}{\cos \beta}$ \\
$H_{3}^{0}$ & $-i \tan \beta$ & $-i \tan \beta$ & & $-i \cot \beta$ & $-i \tan \beta$ \\
\hline
\end{tabular}

$H_{1}^{0}$ and $H_{2}^{0}$ are scalars and $H_{3}^{0}$ is a pseudoscalar. We denote up-type quarks (or leptons) by u and down-type quarks by $d$. Two alternatives, models $I$ and II are described in the text. The parameters $\alpha . \beta$ are defined in eqs. (2) and (3).

In constructing realistic two-higgs doublet models, it is desirable to avoid tree-level higgs-mediated flavor-changing neutral currents [8]. There are two possible types of models which satisfy this constraint:

I. Type I model [7]. $\phi_{1}$ couples to both up-type and down-type quarks and leptons; $\phi_{2}$ does not couple to fermions. The resulting $\mathrm{H}^{0} \mathrm{f} \overline{\mathrm{f}}$ couplings are displayed in table 1 .

2. Type II model $[9,10] . \quad \phi_{1}$ couples to down-type quarks and leptons only and $\phi_{2}$ couples to up-type quarks only. This is the structure required by Peccei-Quinn models [9] and for the supersymmetric extension of the standard model [11]. The resulting $\mathbf{H}^{0}$ f $\bar{f}$ couplings are displayed in table 1.

In order to explain eq. (1c), the $\mathrm{H}^{0} \mathrm{~b} \overline{\mathrm{b}}$ coupling must be enhanced by a factor of order 10 in amplitude. This requires $\cos \beta \ll 1$ (in both models I and II) which implies $v_{2} \gg v_{1}$. However, given this requirement, the two models differ dramatically in their predicted couplings of $\mathbf{H}^{0}$ to up-type quarks. Model II predicts that $\mathrm{H}^{0} \mathrm{c} \overline{\mathrm{c}}$ couplings are severely suppressed compared to couplings with down-type quarks, whereas model I predicts that such couplings are also enhanced. It follows that model II predicts that $\mathrm{H}^{0} \rightarrow \tau^{+} \tau^{-}$is the dominant decay channel of $\mathrm{H}^{0}$ into fermion pairs, whereas in model I,

$$
\left.\frac{\Gamma\left(\mathrm{H}^{0} \rightarrow \mathrm{c} \overline{\mathrm{c}}\right)}{\Gamma\left(\mathrm{H}^{0} \rightarrow \tau^{+} \tau^{-}\right)} \approx \frac{3 m_{\mathrm{c}}^{2}}{m_{\tau}^{2}} \approx 2 \quad \text { (type } \mathrm{I}\right) .
$$

Based on eq. (1d), we see that model II is apparently ruled out; in addition the branching ratio for $\tau^{+} \tau^{-}$must be large enough to satisfy eq. (4) if any simple model is to survive.

Note that in both models we have chosen $\phi_{1}$ to be the higgs doublet which couples to down-type quarks and leptons. In principle, we can define two additional models 
in which the Higgs coupling to quarks remain the same as above but the charged leptons couple to $\phi_{2}$ [12]. In these models when $v_{2} \gg v_{1}$ the $\mathrm{H}^{0} \tau^{+} \boldsymbol{\tau}^{-}$coupling is suppressed. Without the $\tau^{+} \tau^{-}$mode it will be difficult to argue for the Higgs interpretation so we do not consider such models further.

Before proceeding, it is important to comment on the observation displayed in eq. (1e). In the standard picture based on the Wilczek mechanism [6], $\Upsilon, \Gamma^{\prime} \rightarrow \gamma+\mathrm{H}^{0}$ is proportional to $|\psi(0)|^{2}$ where $\psi(r)$ is the appropriate bound-state wave function. Allowing for the different phase space for the two decays leads to $\operatorname{BR}\left(\Upsilon^{\prime} \rightarrow\right.$ $\left.\gamma+\mathrm{H}^{0}\right) / \mathrm{BR}\left(\Upsilon \rightarrow \gamma+\mathrm{H}^{0}\right) \approx 0.76 \pm 0.05$ which is in conflict with eq. (1e). Two groups $[4,5]$ have carefully analyzed this apparent problem. They correctly noted that the prediction just mentioned ignores important mixing effects [7,13] among the produced Higgs boson and $b \bar{b}$ bound states with the same quantum numbers as the emitted higgs (either $0^{++}$or $0^{-+}$). The result of their detailed analysis is quite interesting. We quote the results of ref. [5]:

$$
\frac{\operatorname{BR}\left(\Upsilon^{\prime} \rightarrow \gamma+\zeta\right)}{\operatorname{BR}(\Upsilon \rightarrow \gamma+\zeta)}=0.76 \pm 0.05 \times \begin{cases}0.52, & \zeta=0^{++} \\ 0.97, & \zeta=0^{-+}\end{cases}
$$

where the possible error due to neglected relativistic effects and coupled channel effects has been omitted. The stated conclusion of refs. $[4,5]$ is that eq. (1e) cannot be consistent with a pseudoscalar $\zeta$, but may be consistent with a scalar $\zeta$. If we wish to interpret $\zeta$ as a scalar higgs [5], the $\mathrm{H}^{0} \mathrm{~b} \overline{\mathrm{b}}$ vertex must be enhanced by a factor of about 12 (once the mixing effects have been properly included). With no loss of generality, we will henceforth identify the $\zeta(8.3)$ with $H_{1}^{0}$. From table 1 , this implies that $\cos \alpha / \cos \beta=12$, or $v_{2} / v_{1} \geqslant 12$. Note that the parameters $\alpha$ and $\beta$ are not separately known until additional information is obtained. The coupling [14] to gauge bosons also depends on $\alpha$ and $\beta$ and will allow them to be separately measured. For example, in both type I and type II models, $H_{1}^{0}$ couples to $\mathrm{W}^{+} \mathrm{W}^{-}$ and $Z^{0} Z^{0}$ with a factor $\cos (\alpha-\beta)$ and $H_{2}^{0}$ with $\sin (\alpha-\beta)$. Perhaps in $\overline{\mathrm{p}} \rightarrow \mathrm{W}^{ \pm} \zeta \mathrm{x}$ or $\mathrm{e}^{+} \mathrm{e}^{-} \rightarrow \mathrm{Z} \zeta \mathrm{X}$ this coupling can be observed. Without further information one cannot say whether the coupling to $\zeta(8.3)$ is significantly suppressed.

We shall proceed to consider the implications of assuming that the $\zeta(8.3)$ is a $0^{\cdots}$ Higgs boson and $v_{2} / v_{1} \geq 12$. From our discussion above, the data appear to rule out model II and favor model I. This observation would apparently rule out supersymmetric models whose Higgs sector is necessarily that of model II! We shall see below under what circumstances such a conclusion is warranted.

\section{The $\mathbf{H}^{0} \rightarrow$ gg decay rate}

In model II, the decay $\mathrm{H}^{0} \rightarrow \tau^{+} \tau^{-}$would dominate all other $\mathrm{H}^{0}$ decays into fermion pairs. In order for this to be consistent with eq. (1d), we must account for the high multiplicity events with gluon pairs, which requires $B R\left(H^{0} \rightarrow g g\right)>$ 
$\mathrm{BR}\left(\mathrm{H}^{0} \rightarrow \tau^{+} \tau^{-}\right)$. Is this possible? The decay $\mathrm{H}^{0} \rightarrow \mathrm{gg}$ is a one-loop process: the relevant Feynman diagram is the triangle graph with $\mathrm{H}^{0}$ and the gluons attached at the vertices, as was first noted by Wilczek [6]. Inside the triangle, any colored object which couples at tree level to the $\mathrm{H}^{0}$ is allowed. When the triangle consists of colored fermions, this graph has a wonderful property - namely, for $m_{\mathrm{f}} \gg m_{\mathrm{H}}$, the amplitude for $\mathrm{H}^{0} \rightarrow \mathrm{gg}$ is independent of $m_{\mathrm{l}}$. Thus, in the standard model with one higgs doublet $[13,15,16]$,

$$
\frac{\Gamma(\mathrm{H} \rightarrow \mathrm{gg})}{\Gamma\left(\mathrm{H} \rightarrow \tau^{+} \tau^{-}\right)}=\frac{\alpha_{\mathrm{s}}^{2} m_{\mathrm{H}}^{2}|I|^{2}}{9 \pi^{2} m_{\tau}^{2}}\left(1-\frac{4 m_{\tau}^{2}}{m_{\mathrm{H}}^{2}}\right)^{-3 / 2},
$$

where $I$ is a complex number obtained by summing over all possible particles in the triangle. If only one fermion in the loop were counted and $m_{\mathrm{f}} \gg m_{\mathrm{H}}$, then, $I=1$. By studying the exact expression for $I$ (see, e.g., ref. [16]), one learns that $|I|$ is roughly equal to the number of quarks with mass greater than $\frac{1}{2} m_{\mathrm{H}}$. More precisely, if $m_{\mathrm{H}}=83 \mathrm{GeV}$, one finds after summing over the six flavors of quarks (and taking $\left.\alpha_{\mathrm{s}}=0.16\right)|I|^{2}=\left|\sum_{i=1}^{6} I_{i}\right|^{2} \approx 6.62$. The sum is dominated by the t-quark $(I \approx 1)$, b-quark $(I \approx 1.35)$ and a nearly pure imaginary contribution from the c-quark $(I \approx i)$; the lighter quark contributions are negligible. This leads to the result $\Gamma(\mathrm{H}(8.3) \rightarrow \mathrm{gg}) / \Gamma\left(\mathrm{H}(8.3) \rightarrow \tau^{+} \tau^{-}\right) \approx 0.06$.

Consider the two-higgs doublet models. In model $\mathrm{I}$, the $\mathrm{H}^{0} \mathrm{f} \overline{\mathrm{f}}$ coupling is uniformly enhanced for both u-type and d-type quarks and leptons. Hence, the one-higgs doublet result given by eq. (6) remains unchanged (since both numerator and denominator of eq. (6) are enhanced equally). Therefore, using

$$
\Gamma\left(\mathrm{H}^{0} \rightarrow \tau^{+} \tau^{-}\right)=\frac{\sqrt{2} G_{\mathrm{F}} m_{\mathrm{H}} m_{\tau}^{2}}{8 \pi}\left(1-\frac{4 m_{\tau}^{2}}{m_{\mathrm{H}}^{2}}\right)^{3 / 2}\left(\frac{\cos \alpha}{\cos \beta}\right)^{2}
$$

we find that $\Gamma\left(\mathrm{H}^{0} \rightarrow \mathrm{gg}\right) \approx 100 \mathrm{keV}$.

In model II, only the $\mathrm{H}^{0} \mathrm{dd}$ type couplings are enhanced. Thus, effectively, only d-type quarks contribute appreciably to the loop in $\mathrm{H}^{0} \rightarrow \mathrm{gg}$. If we ignore up-type quarks entirely, we find for three generations of quarks $|I|^{2} \approx 1.6$. More precisely, we may consider all quark flavors using the appropriate factors listed in table 1 . For example, for $H_{1}^{0} \rightarrow \mathrm{gg}$, using $\cos \alpha / \cos \beta=12$ and allowing for $|\cos \alpha| \leqslant 1$, we find that $1.4 \leq|I|^{2} \leq 1.8$. Thus, in model Il, we find $\Gamma(\mathrm{H}(8.3) \rightarrow \mathrm{gg}) / \Gamma\left(\mathrm{H}(8.3) \rightarrow \tau^{+} \tau\right)$ $\approx 0.015-0.02$. Thus, in the minimal two-higgs extension corresponding to model II, the gg decay of the Higgs boson cannot dominate. In this circumstance, we may rule out model II based on eq. (1d).

One can imagine increasing $\Gamma\left(\mathrm{H}^{0} \rightarrow \mathrm{gg}\right)$ by having additional particles inside the $\mathrm{H}^{0} \rightarrow \mathrm{gg}$ triangle graph. However, an enhanced rate for $\mathrm{H}^{0} \rightarrow$ gg would necessarily imply an enhanced production rate for $\mathrm{H}^{0}$ via two-gluon fusion at hac. . .achines 


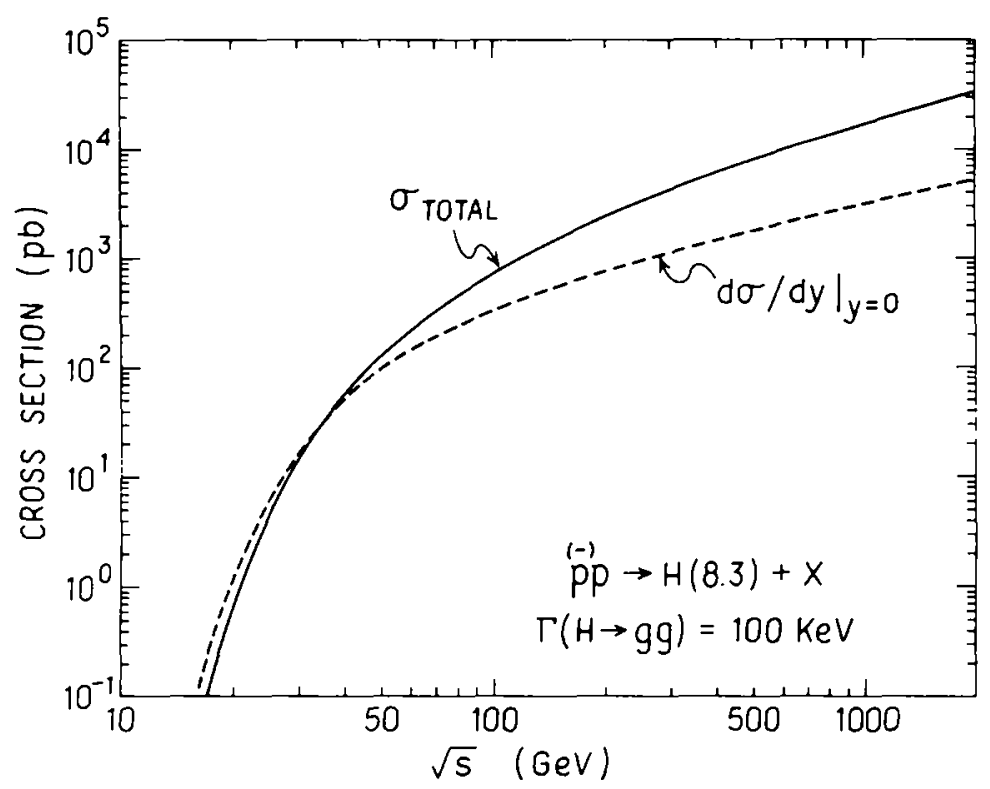

Fig. 1. Production cross section and $\mathrm{d} \sigma / \mathrm{d} y$ at $y=0$ for a Higgs boson with $m_{\mathrm{H}}=8.3 \mathrm{GeV} / \mathrm{c}^{2}$ and $\Gamma\left(\mathrm{H}^{0} \rightarrow \mathrm{gg}\right)=100 \mathrm{keV}$, in $\mathrm{p}^{ \pm} \mathrm{p} \rightarrow \mathrm{H}^{0}+\mathrm{X}$ for $10 \leqslant \sqrt{\mathrm{s}} \leqslant 2000 \mathrm{GeV}$. The curves are obtained from eq. (8) using EHLQ structure functions (ref. [35]) and $A=290 \mathrm{MeV}$. The cross section is proportional to $\Gamma\left(\mathrm{H}^{0} \rightarrow \mathrm{gg}\right)$. One must multiply the above curve by $B_{\mu \mu}$ to obtain the Higgs contribution to $\mathrm{p}^{\circ} \mathrm{p} \rightarrow \mu^{\circ} \mu$ $+\mathrm{X}$. For $\sqrt{s} \leq 100 \mathrm{GcV}$ the variation with different structure functions [35] can be as much as a factor of two: at larger $\sqrt{s}$ there is less dependence on the structure functions.

(as noted by Glashow and Machacek [3]). The standard parton model computation gives $[15]^{\star}$

$$
\frac{\mathrm{d} \sigma}{\mathrm{d} y}=\frac{\pi^{2} \Gamma\left(\mathrm{H}^{0} \rightarrow \mathrm{gg}\right)}{8 m_{\mathrm{H}}^{3}} \tau G\left(\tau^{1 / 2} \mathrm{e}^{y}\right) G\left(\tau^{1 / 2} \mathrm{e}^{y}\right),
$$

where $\tau \equiv m_{\mathrm{H}}^{2} / s, y$ is the Higgs boson rapidity and $G$ is the gluon distribution function. The cross section for $\mathrm{p}^{ \pm} \mathrm{p} \rightarrow \mathrm{H}^{0}+\mathrm{X}$ is shown in fig. 1 , where we have assumed that $\Gamma\left(\mathrm{H}^{0} \rightarrow \mathrm{gg}\right)=100 \mathrm{keV}$ (which is the prediction of model $\mathrm{I}$ ). Using eq. (8), we see that the graph in fig. 1 scales with $\Gamma\left(\mathrm{H}^{0} \rightarrow \mathrm{gg}\right)$.

We now can check whether it is possible to observe a signal in $\mathrm{p}^{\prime} \mathrm{p} \rightarrow \mu^{+} \mu^{-}+\mathrm{X}$. From eq. (7) $\Gamma\left(\mathrm{H}^{0} \rightarrow \mu^{+} \mu^{-}\right)=\left(m_{\mu} / m_{\tau}\right)^{2} \Gamma\left(\mathrm{H}^{0} \rightarrow \tau^{+} \tau^{-}\right)=9 \mathrm{keV}$. Defining $B_{\mu \mu} \equiv$

* The contribution of the subprocess $q \bar{q} \rightarrow H^{0}$ can also be added to eq. ( 8 ). This contribution is obtained from the expression of eq. (8) by replacing $\frac{1}{8} I^{2}\left(\mathrm{H}^{0} \rightarrow \mathrm{gg}\right)$ by ${ }_{9}^{4} I^{2}\left(\mathrm{H}^{0} \rightarrow \mathrm{q} \bar{q}\right)$ (assuming $\left.m_{G} \ll m_{H}\right)$, and by replacing $G$ with the appropriate quark distribution function. However, despite the fact that the $\mathrm{H}^{0} \mathrm{q} \overline{\mathrm{q}}$ couplings are enhanced, $\Gamma\left(\mathrm{H}^{0} \rightarrow \mathrm{q} \overline{\mathrm{q}}\right)$ is still proportional to $m_{\mathrm{q}}^{2}$. Since only the light quarks in the proton are appreciable, the overall contribution of the $q \bar{q} \rightarrow \mathrm{H}^{0}$ subprocess is negligible. 
$\Gamma\left(\mathrm{H}^{0} \rightarrow \mu^{+} \mu^{-}\right) / \Gamma\left(\mathrm{H}^{0} \rightarrow\right.$ all $)$, then by the scaling properties of eq. (8), we obtain

$$
\left.B_{\mu \mu} \frac{\mathrm{d} \sigma}{\mathrm{d} y}\right|_{y=0} \approx 0.8 \frac{\Gamma\left(\mathrm{H}^{0}(8.3) \rightarrow \mathrm{gg}\right)}{\Gamma_{\mathrm{H}}} \mathrm{pb}, \quad \text { at } p_{\mathrm{LAB}}=400 \mathrm{GeV} / c
$$

where $\Gamma_{\mathrm{H}}$ is the total Higgs boson width.

This can be compared to $B_{\mu \mu} \mathrm{do} /\left.\mathrm{d} y\right|_{y=0}=0.30 \pm 0.01 \pm 0.05 \mathrm{pb}$ at $p_{\mathrm{LAB}}=400$ $\mathrm{GeV} / c$ observed at the $\Upsilon$-peak [17]. Using the same data, we estimate that $B_{\mu \mu} \mathrm{do} /\left.\mathrm{d} y\left(\mathrm{pp} \rightarrow \mathrm{H}^{0}(8.3) \rightarrow \mu^{+} \mu^{-}\right)\right|_{y-0} \leq 0.05 \mathrm{pb}$. From eq. (9), this implies for any theory that

$$
\frac{\Gamma\left(\mathrm{H}^{0} \rightarrow \mathrm{gg}\right)}{\Gamma_{\mathrm{H}}} \leq \frac{1}{16}
$$

In model I, we may use eq. (10) to get a limit on $\Gamma\left(\mathrm{H}^{0} \rightarrow \mathrm{gg}\right)$. From eqs. (4) and (7), it follows that $\sum_{\mathrm{f}} \Gamma\left(\mathrm{H}^{0} \rightarrow \mathrm{ff}\right) \approx 6 \mathrm{MeV}$ which implies that $\Gamma\left(\mathrm{H}^{0} \rightarrow \mathrm{gg}\right) \leqslant 400 \mathrm{KeV}$. In models with more than two higgs doublets, eq. (10) is still valid, but now the decay rates into fermion pairs may be altered. To be general, define $x \equiv \Gamma\left(H^{0} \rightarrow\right.$ $\mathrm{gg}) / \Sigma_{\mathrm{f}} \Gamma\left(\mathrm{H}^{0} \rightarrow \mathrm{ff}\right)$. Then, eq. (10) implies that $x \leqslant 0.07$. We conclude that the gluon decay rate cannot be an appreciable fraction of the total Higgs boson width in any model.

The measurement of the width of the $\zeta(8.3)$ is of extreme importance. Using the current upper limit obtained by the Crystal Ball (eq. (1b)) and the results of eq. (10), we find $\Gamma\left(\mathrm{H}^{0} \rightarrow \mathrm{gg}\right) \leqslant 5 \mathrm{MeV}$, independent of the model assumptions. If this bound can be tightened, important constraints can be obtained on possible heavier-colored fermions which couple to the Higgs bosons. For example, in model I, we found above that $\Gamma\left(\mathrm{H}^{0} \rightarrow \mathrm{gg}\right) \leq 400 \mathrm{keV}$. But already with three generations of quarks $\Gamma\left(\mathrm{H}^{0} \rightarrow \mathrm{gg}\right) \approx 100 \mathrm{keV}$. If we add a fourth generation, the width is raised to $\Gamma\left(\mathrm{H}^{0} \rightarrow \mathrm{gg}\right) \approx 300 \mathrm{keV}$. Adding a fifth generation violates the bound. Hence, in a two-higgs doublet model of type I, we already know that there perhaps can be a fourth generation but no more. The basic assumption one makes in this analysis is that the coupling pattern of the lighter generation of quarks to the Higgs bosons is maintained for any heavier fermions, providing a uniform enhancement of all fermion couplings. However, in more complicated models, eq. (10) must still be satisfied. Finally, if a larger $\alpha_{\mathrm{s}}$ is used the constraints are a little tighter.

\section{Implications for supersymmetric models}

In the minimal supersymmetric extension of the standard model (for a review and extensive set of references see [18]), the model consists of two higgs doublets and is necessarily a type II model. In sect. 3, we argued that type II models cannot be compatible with the data on $\zeta$. Therefore, if the $\zeta(8.3)$ is a Higgs boson, the minimal supersymmetric extension of the standard model is wrong. 
Does this mean that low-energy supersymmetry cannot be compatible with the Higgs interpretation of the $\zeta(8.3)$ ? The answer is no, although we must add additional Higgs doublets; the result is not very attractive considering that part of the motivation for supersymmetry is to make the Higgs sector more natural. For example, we can construct a supersymmetric analog of model I. Introduce a model with four Higgs doublets. Two of the Higgs doublets (say $\phi_{1}$ and $\phi_{2}$ ) couple to quarks and leptons in the manner of model II. The other two Higgs doublets ( $\phi_{3}$ and $\left.\phi_{4}\right)$ are totally decoupled from the quarks and leptons (in the same way that $\phi_{2}$ did not couple to quarks in model I). If we let $v_{i}$ be the vacuum expectation values of the neutral components of the $\phi_{i}$, we choose $v_{1}, v_{2} \ll v_{3}, v_{4}$. The $v_{i}$ are normalized such that $\sum v_{i}^{2} \approx 250 \mathrm{GeV}$. In such a model, the $\mathrm{H}^{0} \mathrm{~d} \overline{\mathrm{d}}$ couplings are enhanced by a factor $\left(\sum v_{i}^{2}\right)^{1 / 2} / v_{1}$ and the $\mathrm{H}^{0} \mathrm{u} \overline{\mathrm{u}}$ couplings are enhanced by a factor $\left(\sum v_{i}^{2}\right)^{1 / 2} / v_{2}$. The features of this model then are quite similar to model I and are consistent with the observed data ${ }^{\star}$.

The above model is rather contrived, while a supersymmetric model with a minimal Higgs structure is quite economical. However, there are some indications that a larger Higgs sector in supersymmetric models is needed. For example, in a supersymmetric SU(5) grand unified model, the minimal Higgs sector (two 5's) leads to a negligible cosmological baryon asymmetry in conflict with observation. One needs four Higgs 5's in order to obtain a sufficient amount of baryon asymmetry [19]. This, of course, does not imply that four light weak doublets of Higgs bosons will survive in the effective low-energy theory. But, it provides an example where a non-minimal Higgs multiplet structure is required. Thus, non-minimality is not a reason to reject the supersymmetric approach, but it may have to be included in future model building.

It is also worth noting that simple predictions in the minimal supersymmetric model with two Higgs doublets need not be satisfied in more general models. For example, in the minimal model with two Higgs doublets and $v_{2} \ll v_{1}$, one finds $[11,20,21]$ that the pseudoscalar $\mathrm{H}_{3}^{0}$ is nearly degenerate with the scalar $\mathrm{H}^{0}(8.3)$. Such a feature need not be true in models which contain Higgs singlet fields in addition to Higgs doublets [21], and it is not known whether it holds when extra doublets (but no singlet) are present. It will not be easy to test experimentally for the presence of more than two Higgs doublets. The cleanest signal would probably be the existence of more than one charged Higgs boson.

The discussion regarding supersymmetric models above has been made independently of specific model building. One can now ask whether one can build a sensible model based on the low-energy supergravity approach (for a review of the low-

* One could modify the above four-doublet model in another way. If one coupled $\phi_{1}$ and $\phi_{2}$ to quarks and $\phi_{3}$ and $\phi_{4}$ to leptons, the enhancement of the $\mathrm{H}^{0} \mathrm{~b} \overline{\mathrm{b}}$ and $\mathrm{H}^{0} \tau^{+} \tau^{-}$couplings would be independent. One could then choose the parameters to suppress the $\mathrm{H}^{0} \tau^{1} \tau^{-}$couplings relative to the $\mathrm{H}^{0} \mathrm{~d} \overline{\mathrm{d}}$ couplings. We find such a model quite artificial. Furthermore, if the $\tau^{+} \tau^{-}$decay mode of the $\zeta(8.3)$ is not being observed. it will remove any attractiveness of the Higgs interpretation of the $\zeta$. 
energy supergravity approach see [22]) with (at least) four light Higgs doublets and $v_{2} / v_{1} \geq 12$. The constraints are quite severe. For example, in the minimal model of ref. [23], $v_{2} / v_{1} \geq 6$ implies the existence of a chargino (an appropriate mixture of wino and higgsino) with mass less than $19 \mathrm{GeV}$, in contradiction with current experimental limits [18]. Further analysis will be required to see if a supergravity model can be constructed which is consistent with the required constraints on particle masses.

Even in supersymmetric models with non-minimal Higgs sectors, we must check that the rate for $\mathrm{H}^{0} \rightarrow \mathrm{gg}$ is not too large. In supersymmetric models, when computing the triangle graphs for $\mathrm{H}^{0} \rightarrow \mathrm{gg}$, the scalar quarks as well as the quarks must be included in the triangle. In general this will increase Higgs production cross sections in supersymmetric theories. The relevant Feynman rules have been discussed in ref. [21]. Here, we assume that three generations of scalar quarks are degenerate in mass (with common mass $\tilde{M}$ ), and we neglect effects arising from supersymmetry breaking. The relevant vertices are then [21]

$$
\begin{array}{ll}
H_{1}^{0} \tilde{u}_{\mathrm{L}} \tilde{u}_{\mathrm{L}}: \quad \frac{g m_{\mathrm{Z}}}{\cos \theta_{\mathrm{w}}}\left(\frac{1}{2}-\frac{2}{3} \sin ^{2} \theta_{\mathrm{w}}\right) \cos (\alpha+\beta)+\frac{g m_{\mathrm{u}}^{2}}{m_{\mathrm{w}}} \frac{\sin \alpha}{\sin \beta}, \\
H_{1}^{0} \tilde{u}_{\mathrm{R}} \tilde{u}_{\mathrm{R}}: \quad \frac{g m_{\mathrm{Z}}}{\cos \theta_{\mathrm{w}}} \frac{2}{3} \sin ^{2} \theta_{\mathrm{w}} \cos (\alpha+\beta)+\frac{g m_{\mathrm{u}}^{2}}{m_{\mathrm{w}}} \frac{\sin \alpha}{\sin \beta}, \\
H_{1}^{0} \tilde{d}_{\mathrm{l}} \tilde{d}_{\mathrm{L}}: \quad \frac{-g m_{\mathrm{Z}}}{\cos \theta_{\mathrm{w}}}\left(\frac{1}{2}-\frac{1}{3} \sin ^{2} \theta_{\mathrm{w}}\right) \cos (\alpha+\beta)+\frac{g m_{\mathrm{d}}^{2}}{m_{\mathrm{w}}} \frac{\cos \alpha}{\cos \beta}, \\
H_{1}^{0} \tilde{d}_{\mathrm{R}} \tilde{d}_{\mathrm{R}}: \quad \frac{-g m_{\mathrm{Z}}}{\cos \theta_{\mathrm{w}}} \frac{1}{3} \sin ^{2} \theta_{\mathrm{w}} \cos (\alpha+\beta)+\frac{g m_{\mathrm{d}}^{2}}{m_{\mathrm{w}}} \frac{\cos \alpha}{\cos \beta} .
\end{array}
$$

The $H_{2}^{0}$ couplings are obtained by the following replacements: $\sin \alpha \rightarrow \cos \alpha, \cos \alpha$ $\rightarrow-\sin \alpha$ and $\cos (\alpha+\beta) \rightarrow-\sin (\alpha+\beta)$. Note that the mass of the quark appears in eq. (11) and not the mass of the scalar quark, $\tilde{M}$. Adding up the contributions from one generation of scalar quarks, we see that the terms in eq. (11) proportional to $m_{\mathrm{z}}$ exactly cancel. When the remaining terms are included, we find, making use of Vainshtein et al. [16], that $|I|$ in eq. (6) is modified by the replacement

$$
I=I_{\mathrm{q}}+\frac{1}{2} \sum_{\mathrm{q}} \frac{m_{\mathrm{q}}^{2}}{\bar{M}^{2}},
$$

where $I_{\mathrm{q}}$ is the contribution of the quark loops, and the sum over quarks $\mathrm{q}$ has already taken into account the effects of both $\tilde{q}_{L}$ and $\tilde{q}_{R}$ loops. Note that in the supersymmetric limit where $m_{\mathrm{q}}=\tilde{M}$, we see that (asymptotically, where $\tilde{M} \gg m_{\mathrm{H}}$ ) each scalar-quark loop (taking $\tilde{q}_{\mathrm{L}}$ and $\tilde{q}_{\mathrm{R}}$ separately) counts as $\frac{1}{4}$ of the quark loop. 
If we apply eq. (12) to a three-generation minimal supersymmetric model, the effect of the scalar quarks on $\Gamma\left(\mathrm{H}^{0} \rightarrow \mathrm{gg}\right)$ is negligible. According to eq. (11), the surviving terms which are enhanced (recall that $\cos \alpha / \cos \beta \approx 12$ by assumption) are the down-type scalar quarks. But, recent analysis [24-26] of UAl data suggests that $m_{\mathrm{b}} / \tilde{M} \leqslant 0.1$ which indeed leads to a negligible modification of $I_{\mathrm{q}}$ in eq. (12). If we relax the assumption of degenerate-mass scalar quarks, the terms proportional to $m_{z}$ in eq. (11) can contribute. But even in the most favorable of circumstances, the effects of the scalar-quark loops remain small. In a model with four generations, it is possible to have $m_{\mathrm{q}} \approx \tilde{M}$ for fourth-generation quarks and their partners. Then by eq. (12), each $\tilde{q}_{\mathrm{L}}$ and $\tilde{q}_{\mathrm{R}}$ counts for one-half of a fermion inside the $\mathrm{H}^{0} \rightarrow \mathrm{gg}$ triangle. Thus, in this case the scalar quarks could make an observable difference. In principle, an accurate measurement of the total $\mathrm{H}^{0}$ width in conjunction with the bound of eq. (10) could rule out this possibility.

\section{Implications for other approaches beyond the standard model}

We have already remarked at the end of sect. 3 that under certain assumptions, one could rule out the possible existence of additional heavy generations of quarks beyond a hypothetical fourth generation.

One could imagine something more exotic. Suppose that fermions with exotic color quantum numbers exist (as suggested by some composite models [27]). These fermions could couple to the Higgs bosons and therefore substantially boost the rate for $\mathbf{H}^{0} \rightarrow \mathrm{gg}$. For comparison, the color factors relevant for $\mathrm{H}^{0} \rightarrow \mathrm{gg}$ via a triangle of colored Dirac fermions in the representation $R$ is given by $\left(\operatorname{Tr}\left(T^{a} T^{b}\right)\right)^{2}=\left(T_{R} \delta^{a b}\right)^{2}$ $=8 T_{R}^{2}$. For SU(3) representations $\underset{\sim}{3}, \underset{2}{6}$ and $\underset{\sim}{8}, T_{R}=\frac{1}{2}, \frac{5}{2}, 3$ respectively ${ }^{\star}$. Thus, the contribution of one color octet heavy fermion would be 36 times larger than that of a color triplet heavy quark. Higher representations would be even more significant. The contribution of such exotics to the $\mathbf{H}^{0} \rightarrow \mathrm{gg}$ triangle are severely limited. For example, in model I we may write

$$
\left(\frac{\Gamma(\mathrm{H} \rightarrow \mathrm{gg})}{0.11 \mathrm{keV}}\right)^{1 / 2} \approx 31+12 N_{3}+5 \Lambda_{6} N_{6}+6 \Lambda_{8} N_{8}+\cdots,
$$

31 is the contribution of the three known generations, and $N_{3}$ is the number of quarks beyond the t-quark, each one assumed to have couplings to the Higgs bosons enhanced by a factor of 12 . Further terms are due to $N_{R}$ hypothetical color exotics in the representation $R$ (with mass $m$ ) which couple to the Higgs boson with strength $\left(G_{\mathrm{F}} \sqrt{2}\right)^{1 / 2} \Lambda_{R} m$. A better bound on $\Gamma(\mathrm{H} \rightarrow \mathrm{gg})$ will significantly constrain the $N_{i}$ and $\Lambda_{i}$. As noted above, in model $\mathrm{I} \Gamma\left(\mathrm{H}^{0} \rightarrow \mathrm{gg}\right) \leqslant 400 \mathrm{keV}$, so $N_{3} \leqslant 2$ and $N_{6}, N_{8}=0$ if $\Lambda_{6}$ and $\Lambda_{8}$ are enhanced similarly to $\Lambda_{3}=12$.

\footnotetext{
* For the SU(3) representation denoted by $\left(a_{1}, a_{2}\right)$, the dimension is given by $d=\frac{1}{2}\left(a_{1}+1\right)\left(a_{2}+1\right)$ $\left(a_{1}+a_{2}+1\right)$ and the index is given by $2 T_{R}={ }_{12}^{1} d\left(a_{1}^{2}+a_{2}^{2}+3 a_{1}+3 a_{2}+a_{1} a_{2}\right)$. Note that there is misprint in the formula for the index given in ref. [28].
} 
Lastly, we briefly mention the implication of a Higgs interpretation of the $\zeta(8.3)$ for technicolor and composite Higgs theories. In technicolor models [29,30], $0^{++}$ scalar bosons tend to be very heavy (above $100 \mathrm{GeV}$ in mass), whereas light $0^{-+}$ scalar bosons ("technipions") are predicted in many models. Could the $\zeta$ be a technipion, given that the arguments of refs. $[4,5]$ strongly suggest that the $\zeta$ cannot be a $0^{-+}$boson? This question has been discussed previously [31] in the context of the $\xi(2.2)$. The answer is somewhat subtle. Technipions have $0^{--}$couplings in their interaction with gauge bosons. But technipion interactions with fermions depend on the unknown dynamics of the extended technicolor sector of the theory. Scalar couplings of technipions to a pair of quarks may develop $[30,31]$ if either (a) there is a CP violation or (b) the technipion is made out of a pair of non-identical techniquarks. In case (b), one can have scalar couplings in a manner analogous to the $\mathrm{K}_{\mathrm{L}}^{0}-\mathrm{K}_{\mathrm{s}}^{0}$ system in QCD where the "pseudoscalar" $\mathrm{K}_{\mathrm{s}}^{0}$ is in fact a $C P$-even scalar boson. In this case, one predicts [31] a CP-odd technipion (analogous to the $\mathrm{K}_{\mathrm{L}}^{0}$ ) which can also be searched for in $\gamma \rightarrow \gamma+\mathrm{X}$. In case (a), one would have to make a detailed study of the quantum numbers of the $\zeta$ and search for an admixture of $0^{-}$and $0^{+}$quantum numbers. This requires one to measure the polarization of the photon in $r \rightarrow \gamma+\zeta(8.3)$ which can best be done by having the photon convert into a lepton pair. The necessary theoretical analysis has been performed in refs. [32, 33]; clearly a large sample of $\Upsilon$ would be required $\left(>10^{6}\right)$ in order to perform such a test experimentally. The conclusion here is that although the technicolor approach cannot be ruled out, it is clear that the existence of a light scalar Higgs-like particle does not easily coexist with the technicolor scheme. A similar remark is probably appropriate to models of composite higgses (see e.g. refs. [34]). In these models, the scalar higgs tends to be heavier than the W-mass. Such models are hard pressed to explain a light-scalar Higgs state.

\section{Conclusions and discussion}

We have reached a fascinating state of affairs. Theorists have long been dissatisfied with the elementary scalar higgs. Many models have been proposed beyond the standard model which attempt to achieve a more fundamental understanding of the scale of electroweak symmetry breaking. Such approaches include supersymmetry, composite models, technicolor and composite Higgs models. Now, the Crystal Ball collaboration has presented evidence of a new state, the $\zeta(8.3)$, which apparently shows some of the behavior expected of a Higgs boson. If the $\zeta$ is a Higgs particle, it must be a scalar (not a pseudoscalar) in a multi-higgs doublet model with enhanced couplings to down-type fermions and also enhanced couplings to up-type quarks. We have demonstrated that its gluon pair branching ratio is small. The existence of such a Higgs boson is a real puzzle in the context of all current models. Technicolor and composite Higgs models do not seem to be compatible with a light-scalar Higgs boson. The minimal supersymmetric extension of the standard model is inconsistent 
with such a Higgs boson since it is not seen to decay entirely into $\tau^{+} \tau^{-}$. (One can, however, construct non-minimal four-higgs doublet supersymmetric models which can satisfy the observed data.) Finally, one can put a bound on the rate for $\mathrm{H}^{0} \rightarrow \mathrm{gg}$, obtaining strong constraints on heavier generations of quarks (only one more sequential family could exist in the simplest two-doublet Higgs model). One also finds restrictions on new heavy exotic-colored fermions which couple to the higgs, as suggested in some composite models. In addition to confirming the $\zeta(8.3)$ signal and the presence of a $\tau^{+} \tau^{-}$mode larger than $\mu^{+} \mu^{-}$and $\mathrm{e}^{+} \mathrm{e}^{-}$in proportion to the masses, it is of great importance to confirm that the dominant decay is c $\bar{c}$ from the presence of the semileptonic decay. If the Higgs interpretation of $\zeta(8.3)$ survives, apparently the masses of gauge bosons and of fermions are generated in largely uncoupled ways, as in the approach of refs. [6,7].

Conversations with Michael Peskin were invaluable in the development of this work. We would also like to thank Jack Gunion, Marc Sher and Henry Tye for useful comments.

\section{References}

[1] C. Peck, et al., DESY/SLAC preprint DESY 84-064 and SLAC-PUB-3380 (1984), paper submitted to the XXII Int. Conf. on High energy physics, Leipzig. July 19-25, 1984

[2] S.-H.H. Tye and C. Rosenfeld, Comell preprint CLNS-84/621 (1984)

[3] S.L. Glashow and M. Machacek, Harvard preprint HUTP-84/A063 (1984); K. Lane, S. Meshkov and F. Wilczek, Santa Barbara preprint NSF-ITP-84-116 (1984)

[4] J. Polchinski, S.R. Sharpe and T. Barnes, Harvard preprint HUTP-84/064 (1984)

[5] J. Pantaleone, M.E. Peskin, and S.-H.H. Tye, SLAC-PUB-3439 and Cornell preprint CLNS-84/622 (1984)

[6] F. Wilczek, Phys. Rev. Lett. 39 (1977) 1304

[7] H.E. Haber, G.L. Kane and T. Sterling, Nucl. Phys. B161 (1979) 493

[8] S.L. Glashow and S. Weinberg, Phys. Rev. D15 (1977) 1958

[9] R. Peccei and H. Quinn, Phys. Rev. D16 (1977) 1791;

S. Weinberg, Phys. Rev. Lett. 40 (1977) 223;

F. Wilczek, Phys. Rev. Lett. 40 (1977) 279

[10] J.F. Donoghue and L.-F. Li, Phys. Rev. D19 (1979) 945; B. McWilliams and L.-F. Li, Nucl. Phys. B179 (1981) 62

[11] K. Inoue, A. Kakuto, H. Komatsu and S. Takeshita, Prog. Theor. Phys. 67 (1982) 1889; 68 (1982) 927

[12] S.-H.H. Tye, invited talk given at Physics in Collision IV Conf., Santa Cruz, August 22-24, 1984

[13] J. Ellis, M.K. Gaillard, D.V. Nanopoulos and C.T. Sachrajda, Phys. Lett. 83B (1979) 339

[14] H. Huffel and G. Pocsik, Z. Phys. C8 (1981) 13:

G. Pocsik and G. Zsigmond. Z. Phys. C10 (1981) 367; Phys. Lett. 112B (1982) 157

[15] H. Georgi, S.L. Glashow, M. Machacek and D.V. Nanopoulos, Phys. Rev. Lett. 40 (1978) 692

[16] A.I. Vainshtein, M.B. Voloshin, V.I. Zakharov and M.A. Shifman, Sov. J. Nucl. Phys. 30 (1979) 711: T.G. Rizzo. Phys. Rev. D22 (1980) 178

[17] K. Ueno et al., Phys. Rev. Lett. 42 (1979) 486

[18] H.E. Haber and G.L. Kane, Michigan preprint UM TH 83-17 (1984). Phys. Reports C. to be published

[19] H.E. Haber, Phys. Rev. D26 (1982) 1317 
[20] R.A. Flores and M. Sher, Ann. of Phys. 148 (1983) 95

[21] J.F. Gunion and H.E. Haber, SLAC-PUB-3404 (1984)

[22] H.P. Nilles, Geneva preprint UGVA-DPT 1983/12-412 (1984). Phys. Reports C, to be published

[23] L. Alvarez-Gaumé, J. Polchinski and M.B. Wise. Nucl. Phys. B221 (1983) 495

[24] J. Ellis and H. Kowalski, DESY 84-045 (1984)

[25] V. Barger, K. Hagiwara and W.-Y. Keung, Wisconsin preprint MAD/PH/183 (1984)

[26] R.M. Barnett, H.E. Haber and G.L. Kane, preprint in preparation

[27] S.F. King, Phys. Lett. 107B (1981) 201: Nucl. Phys. B214 (1983) 36: Phys. Lett. 136B (1984) 371; E.J. Squires, J. Phys. G7 (1981) L47;

H. Harari and N. Seiberg, Nucl. Phys. B204 (1982) 141

[28] J. Patera and D. Sankoff, Tables of branching rules for representations of simple Lic algcbras (University of Montreal Press, Montreal, 1973)

[29] S. Weinberg, Phys. Rev. D13 (1976) 974; D19 (1979) 1277;

L. Susskind, Phys. Rev. D20 (1979) 2619;

E. Farhi and L. Susskind. Phys. Reports $74 \mathrm{C}$ (1981) 277

[30] K. Lane, in Proc. 1982 DPF Summer Study on Elementary particle physics and future facilities, ed. R. Donaldson, R. Gustafson and F. Paige (American Physical Society, New York. 1983) 222

[31] H.E. Haber, SLAC-PUB-3193 (1983)

[32] G.J. Gounaris and A. Nicolaidis, Phys. Lett. 102B (1981) 144; 109B (1982) 221

[33] T. Shimada. Meiji University preprint (1981)

[34] H. Georgi and D.B. Kaplan, Phys. Lett. 136B (1984) 183, 187; Harvard preprint HUTP-84/A034 (1984);

H. Georgi, D.B. Kaplan and P. Galison, Harvard preprint HUTP-84/A018 (1984)

[35] E. Eichten, I. Hinchliffe, K. Lane and C. Quigg, Fermilab-Pub-84/17-T (1984):

D.W. Duke and J.F. Owens, Phys. Rev. D30 (1984) 49 